\title{
Magnetofluidics for Manipulation of Convective Heat Transfer
}

\author{
Majid Hejazian and Nam-Trung Nguyen \\ Queensland Micro and Nanotechnology Centre, Griffith University, Brisbane, Queensland \\ 4111, Australia
}

A B S T R A C T

We report heat transfer manipulation and control in a magnetofluidic device. The device consists of a circular chamber with a heat source on top and is positioned next to a permanent magnet that creates a non-uniform magnetic field. Convective heat transfer was evaluated and compared for three cases: DI-water, ferrofluid, and ferrofluid under the magnetic field. Experimental results indicate enhancement of convective heat transfer with the use of diluted ferrofluid as the working fluid. However, we observed a reduction in outlet temperature and Nusselt number in the presence of a non-uniform magnetic field. In addition, we report a full simulation of transport phenomena in the system explaining the physics of this phenomenon. The simulation results agree with the experimental data, and show the same trends.

Keywords: ferrofluid; heat transfer; magnetic field; CFD; magnetofluidics

\section{Introduction}

Heat transfer enhancement is the main challenge for various applications in electronics and industrial cooling systems. Ferrofluids offer promising advantages for heat transfer augmentation. Ferrofluids are stable suspensions of paramagnetic nanoparticles, typically magnetite, in non-magnetic base fluids such as water and oil. Several studies have reported improvement of thermophysical properties of the base fluid using nanofluids [1-21].

Utilizing paramagnetic ferrofluids in the presence of a magnetic field for the control of heat transfer has attracted growing interest of many researchers recently [22-27]. Using this method in systems with contentious forced convection, fluid flow and thermophysical properties of the ferrofluid can be altered due to the migration of paramagnetic nanoparticles under an external magnetic field. As a result, temperature distribution and convective heat transfer can be controlled locally in a magnetofluidic based device.

Goharkhah et al. recently investigated the laminar forced convective heat transfer of ferrofluid in a long uniformly heated parallel plate channel under an external magnetic field 
[28] [29]. Increasing the flow rate and concentration of the ferrofluid enhanced the convective heat transfer. The authors reported an increase of $16.4 \%$ in heat transfer by the use of ferrofluid and in the absence of a magnetic field. Exposure to a constant and alternating magnetic field achieved an improvement of $24.9 \%$ and $37.3 \%$, respectively. In the absence of the magnetic field, the application of ferrofluid improved the average convective heat transfer up to $13.5 \%$ compared to DI-water. Under a constant and alternating magnetic field, this value grows up to $18.9 \%$ and $31.4 \%$, respectively.

Xuan et al. used the Lattice-Boltzmann method to model forced convection of ferrofluid flow in a microchannel [30]. The authors reported that heat transfer might be enhanced or suppressed if the magnetic field gradient is perpendicular to the main ferrofluid flow. Heat transfer is improved if the magnetic field gradient is parallel to the temperature gradient. Azizian et al. investigated the influence of magnetic field on the convective heat transfer of the laminar ferrofluid flow [31]. The accumulation of paramagnetic nanoparticles leads to an increase in thermal conductivity and consequently heat transfer improvement, but with a penalty in pressure drop. Goharkhah and Ashjaee reported a numerical study on forced convective heat transfer of ferrofluid under an alternating non-uniform magnetic field [32]. Compared to the case with no magnetic field, heat transfer enhancement was observed with the presence of the magnetic field. The pressure drop was also reported to increase with increasing magnetic field strength.

Yarahmadi et al. performed an experimental study on the effect of ferrofluid and a magnetic field on heat transfer in a tube under a constant heat flux [33]. The authors reported that compared with distilled water convective heat transfer improves in the absence of a magnetic field. Furthermore, an oscillatory magnetic field enhances the convective heat transfer through the circular tube, while heat transfer decreased with a constant magnetic field. Lajvardi et al. reported heat transfer enhancement for forced convective ferrofluid flow in a heated copper tube in the presence of magnetic field [34]. The effect of ferrofluid concentrations and magnet position on heat transfer was also examined. Change of thermophysical properties of the ferrofluid under the influence of the applied magnetic field was mentioned as the reason for heat transfer augmentation. Ghofrani et al. reported heat transfer enhancement with a ferrofluid flow inside a circular copper tube in the presence of an alternating magnetic field [35]. The interaction between the magnetic field and the nanoparticles created a complex convection regime that led to heat transfer augmentation. The alternating magnetic field proved to enhance the convective heat transfer rate. Heat 
transfer improved with increasing frequency of the alternating magnetic field and increasing concentration of the ferrofluid. At low Reynolds numbers, a maximum increase of $27.6 \%$ in the convection heat transfer was reported. Recently, Asfer et al. experimentally examined the effect of magnetic field on ferrofluid flow in a circular stainless steel channel [36]. The authors used infrared thermography (IRT) to investigate the temperature profile. The authors reported that the magnetic force could have a positive or negative effect on convective heat transfer coefficient. The transport of heat increases with increasing magnetic field gradient as in case of double-inline arrangement of magnets compared to that of single-inline arrangement of magnets.

Our present work reports a numerical simulation and experimental verification of convective heat transfer of ferrofluid in a microchamber in the presence of an external magnetic field. As reviewed above, the effect of an external magnetic field on forced convective heat transfer has been reported in the literature. Governing equations for momentum, heat and mass transfer were solved using COMSOL to gain a better understanding of the phenomena, which is missing in the literature. In addition, we examine the effect of magnet arrangement, direction of the magnetic field gradient, concentration of the ferrofluid, and flow rate.

\section{Materials and methods}

Figure 1 shows a schematic of the geometry employed in this study. The inlet and outlet channels have a depth of $H=500 \mu \mathrm{m}$ and a width of $W=2 \mathrm{~mm}$. The circular chamber has a depth of $H=500 \mu \mathrm{m}$ and a diameter of $D=6 \mathrm{~mm}$. We used a laser engraving machine (Trotec/Rayjet) to cut the channel through a clear $500 \mu \mathrm{m}$ thick double-sided adhesive tape (source, brand of the tape). The upper wall of the circular chamber was closed with a thermoelectric cooler (TEC1-007031010 KJLP (SHENZHEN) CO.). The remaining area of the channel was bonded to poly(methyl methacrylate) PMMA slides. Two thermocouples (RS Pro K Type Thermocouple, RS Components Pty Ltd) measure the inlet and outlet temperatures. The slots for the inlet, outlet and thermocouples were cut through the upper PMMA slide before bonding to the tape. A neodymium-iron-boron (NdFeB) permanent magnet cube with a volume of $3.2 \mathrm{~mm}^{3}$ and grade of N42 (B222, K\&J Magnetics, Inc.) was placed next to the chamber, in order to study the effect of the magnetic field. The magnetic field magnitude of the individual permanent magnet versus distance from the magnet was measured and reported previously [37]. Diluted water-based ferrofluid (EMG707, Ferrotec) 
was fed into the device as a paramagnetic fluid. The commercial ferrofluid has an initial magnetic susceptibility of 0.12 , a magnetic particle concentration of $2 \%$ vol. and a saturation magnetization of $110 \mathrm{G}$. The ferrofluid was further diluted by deionized water (DI-water) into $20 \%$ vol. concentration. A precision syringe pump (SPM100, SIMTech Microfluidics Foundry) delivers the fluid into the device. The flow rate in this experiment ranges from 50 to $400 \mu \mathrm{L} / \mathrm{min}$.

\section{Numerical analysis}

We perform a full simulation including transport of heat, mass and momentum. The numerical model with COMSOL (COMSOL Inc., USA) simulated the transport phenomena in the circular chamber. The two-dimensional model consists of one inlet, a circular chamber and one outlet. The shallow channel approximation was applied to consider the height of the channels. The fluid flow was considered as an incompressible. Steady-state conditions were applied for the simulation. Three separate domains were modelled: the permanent magnet, the channel and the surrounding PMMA layer. A circular magnetic insulation boundary conditions with a diameter of $100 \mathrm{~mm}$ were applied around the system to bind the magnetic field and to achieve an accurate field distribution.

The laminar flow of diluted ferrofluid inside the chamber is governed by the continuity equation:

$\boldsymbol{\nabla} \cdot\left(\boldsymbol{\rho}_{\mathrm{f}} \boldsymbol{u}_{f}\right)=\mathbf{0}$

and the Navier-Stokes equation:

$\nabla\left[-p_{f} I+\mu_{f}\left(\nabla u_{f}+\left(\nabla u_{f}\right)^{T}\right)\right]-12 \frac{\mu_{f} u_{f}}{H^{2}}+($ M. $\nabla) \mathrm{B}=0$

where $\boldsymbol{u}_{\boldsymbol{f}}$ is the fluid velocity, $\boldsymbol{p}_{\boldsymbol{f}}$ the pressure, $\boldsymbol{\nabla}()^{\boldsymbol{T}}$ the divergence operator, $\boldsymbol{\nabla}()$ the gradient operator, $\boldsymbol{I}$ the identity matrix, $\boldsymbol{\mu}_{\boldsymbol{f}}$ the fluid dynamic viscosity, $\mathbf{B}$ magnetic flux density, $\mathbf{M}$ magnetization vector, and $H$ is the height of the channel. No slip conditions were applied at the walls, and the pressure has no viscous stress at the outlet.

The magnetic field in the absence of electric currents is described by:

$\nabla \cdot\left(\frac{\mu_{0} \mu_{r} \mathrm{M}}{x_{m}}\right)=\mathbf{0}$ 
where $\boldsymbol{\mu}_{\boldsymbol{r}}$ is the relative magnetic permeability and $\boldsymbol{\mu}_{\mathbf{0}}$ is magnetic permeability constant.

The energy equation is formulated as:

$\rho_{f} c_{p f}\left(u_{f} \cdot \nabla \mathrm{T}\right)=k_{f} \nabla^{2} T-\mu_{f} T \frac{\partial \mathrm{M}}{\partial T} \cdot\left(\frac{\left(u_{f} \cdot \nabla\right) \mathrm{M}}{x_{m}}\right)$

where $T$ is the temperature, $\boldsymbol{c}_{\boldsymbol{p} \boldsymbol{f}}$ the heat capacity of the ferrofluid, $\boldsymbol{k}_{\boldsymbol{f}}$ thermal conductivity of ferrofluid, and $\boldsymbol{x}_{\boldsymbol{m}}$ the total magnetic susceptibility of the fluid.

The diffusive/convective transport of a solute is obtained by solving:

$-\nabla \cdot\left(-D \nabla c+c u_{f}\right)=0$

where $c\left(\mathbf{m o l} / \mathbf{m}^{3}\right)$ is the concentration, and $D\left(\mathbf{m}^{2} / \mathbf{s}\right)$ is the diffusion coefficient of the solute.

Using single phase model, the density of the ferrofluid is calculated by:

$\rho_{\mathrm{f}}=(1-\varphi) \rho_{\mathrm{water}}+\varphi \rho_{\mathrm{np}}$

where $\boldsymbol{\rho}_{\mathbf{n p}}$ and $\boldsymbol{\varphi}$ are the density of nanoparticles and volume fraction, respectively. The effective dynamic viscosity of the ferrofluid is estimated as:

$\mu_{\mathrm{f}}=\mu_{\mathrm{water}}\left(\frac{1}{(1+\varphi)^{0.25}}\right)$

The effective thermal conductivity of the ferrofluid was determined by [17]:

$\frac{k_{n f}}{k_{f}}=1+64.7 \phi^{0.7640}\left(\frac{d_{f}}{d_{p}}\right)^{0.3690}\left(\frac{k_{f}}{k_{p}}\right)^{0.7476} \operatorname{Pr}_{T}^{0.9955} \operatorname{Re}_{T}^{1.2321}$

(8)

The Prandt number $\boldsymbol{P} \boldsymbol{r}_{\boldsymbol{T}}$ and the Reynolds number $\boldsymbol{R} \boldsymbol{e}_{\boldsymbol{T}}$ are defined as:

$\operatorname{Pr}_{T}=\frac{\mu_{f}}{\rho_{f} \alpha_{f}}$ 


$$
R e_{T}=\frac{\rho_{f} k_{b} T}{3 \pi \mu_{f}^{2} l_{f}}
$$

where $\boldsymbol{k}_{\boldsymbol{b}}=\mathbf{1 . 3 8 0 7} \times \mathbf{1 0}-\mathbf{2 3} \mathrm{J} / \mathbf{K}$ is the Boltzmann constant, $\boldsymbol{l}_{\boldsymbol{f}}$ and is the mean path of fluid particles given as $0.17 \mathrm{~nm}$. This model considers effects of temperature and nanoparticle size for the temperature ranging from 21 to $70{ }^{\circ} \mathrm{C}$.

The heat capacity of the ferrofluid is evaluated as:

$$
\left(\rho c_{p}\right)_{f f}=(1-\varphi)\left(\rho c_{p}\right)_{f}+\varphi\left(\rho c_{p}\right)_{n p}
$$

where $\boldsymbol{\varphi}$ is volume fraction of the solid particles, and subscripts $\boldsymbol{f}, \boldsymbol{f} \boldsymbol{f}$ and $\boldsymbol{n} \boldsymbol{p}$ stand for base fluid, ferrofluid and magnetic nanoparticles, respectively.

The flow and magnetic fields were coupled first to obtain the velocity field of the ferrofluid under the effect of magnetic field. The diffusive/convective transport of nanoparticles and the energy transport equation were solved using the velocity field of the ferrofluid to achieve concentration and temperature field. Finite element method was employed for the solution of the above equations. Finite element discretization was based on the second-order functions for velocity, quadratic functions for concentration, and linear functions for the pressure and temperature fields. We utilized user controlled meshing in COMSOL, consisting of 98808 domain elements and 1461 boundary elements. The numerical simulation was carried out for seven flow rates at fixed heat flux, magnetic field magnitude and ferrofluid concentration. Figure 2 shows the representative results for magnetic field, velocity field, concentration field, and temperature distribution for the flow rate of $50 \mu \mathrm{L} / \mathrm{min}$ of ferrofluid under the effect of magnetic field.

\section{Results and discussion}

The experiments were performed for a constant heat flux, from the top of the chamber, and a constant magnitude of the magnetic field. We apply different flow rates to vary the strength of hydrodynamic flow. The experiment was carried out with DI-water, diluted ferrofluid, and diluted ferrofluid under the effect of the magnetic field. Our aim is to investigate the effect of the iron oxide nanoparticles to the DI-water, and the effect of magnetic field on heat transfer performance. A relatively high concentration of diluted 
ferrofluid, $20 \%$ vol., was selected to increase the effect of magnetic field on both thermophysical properties and bulk force on convective heat transfer.

Fig. 2 Illustrates the representative simulation results, with ferrofluid and under the effect of magnetic field. The magnitude of magnetic field decays with increasing distance from the permanent magnet. The non-uniform magnetic field distribution is depicted in Fig.2(a). The velocity field inside the chamber is depicted in Fig. 2(b). The accumulation of paramagnetic nanoparticles next to the permanent magnet indicated by the higher concentration can be seen in Fig. 2(c). This phenomenon is caused by the positive magnetophoresis of the magnetite nanoparticles toward the magnet. The movement creates a secondary flow in the direction of magnetic field maxima leading to a longer residence time in the chamber. Fig. 2(d) shows the distribution of the temperature inside the chamber. A higher temperature gradient exists at the centre of the chamber due to the higher velocity, shorter resident time, and more convective heat transfer compared with the areas away from the centre.

Figure 3 compares the outlet temperature obtained from the experiments and the simulation. Three cases were considered: DI-water as the operating fluid, diluted ferrofluid as the operating fluid, and ferrofluid under the effect of magnetic field. The experimental data demonstrate that with an increasing flow rate, the outlet temperature decreases due to the higher convective heat transport. Comparing DI-water and ferrofluid with no magnetic field indicate that the presence of nanoparticles increases the outlet temperature considerably. The main reason for convective heat transfer enhancement with ferrofluid is the improvement of thermophysical properties of a nanofluid, according to equations (8) and (11). In addition, movement of nanoparticles can lead to a stronger energy exchange in the fluid and agitate the thermal boundary layer and consequently increase the temperature gradient between the operating fluid and the heat source. However, the outlet temperature drops significantly to the values even less than that of DI-water with the presence of the magnetic field in the system. This phenomenon is caused by the secondary flow, which is perpendicular to the direction of main flow and increases the residence time of the ferrofluid in the chamber.

According to the experimental data, at flow rates higher than $300 \mu \mathrm{L} / \mathrm{min}$ the outlet temperature approaches that of the case with ferrofluid and no magnetic field. However, this effect is more distinguishable in the simulation, Fig. 3(b). The value of outlet temperature is the highest in the case of ferrofluid and no magnetic field for the whole range of flow rates. By adding the magnetic field to the system, the outlet temperature substantially drops to 
values even lower than that of DI-water. This trend continues for the flow rates as high as 350 $\mu \mathrm{L} / \mathrm{min}$. For flow rates higher than $350 \mu \mathrm{L} / \mathrm{min}$ the hydrodynamic force of the main flow overcomes the effect of the secondary flow and the value of the outlet temperature come close to that of ferrofluid with no magnetic field.

Figure 4 displays the variation of the total Nusselt number $\mathrm{Nu}$ versus Reynolds number Re. Convective heat transfer grows with the increase of flow rate for all operating fluids. According to experimental data (Fig. 4(a)), addition of nanoparticles to DI-water enhances the total convective heat transfer in the device. This improvement is more pronounced with higher Re numbers. The addition of magnetic field to the system at low flow rates, where magnetoconvective secondary flow is significant, has a negative effect on the total convective heat transfer of the device. With a higher Reynolds number Re, the negative effect of secondary flow starts to disappear due to the dominant hydrodynamic force of the main flow. As a result, the Nusselt number $\mathrm{Nu}$ for the case with magnetic field tends to approach the values with ferrofluid and no magnetic field. Figure 4(b) shows the simulations results, which indicate the same effect as in the experiment: the magnetic field leads to trapping of heat in the circular chamber.

\section{Conclusions}

We report magnetic control of heat transfer in a circular chamber experimentally and numerically. We fabricated a microfluidic device for this purpose. Three cases were examined and compared: DI-water, diluted ferrofluid, and diluted ferrofluid in a magnetic field. Using diluted ferrofluid as the operating fluid results in heat transfer augmentation. Experimental data indicate that the presence of a magnetic field causes heat trapping at a relatively low flow rate. In the presence of a magnetic field, paramagnetic nanoparticles move towards the magnet. Convective heat transfer reduction is a result of magnetoconvective secondary flow of the ferrofluid towards the permanent magnet. Increasing flow rate reduces the negative effect of the induced secondary flow. As a result, convective heat transfer and Nu number improves at higher flow rates. A full simulation of the phenomenon was performed, considering momentum, heat and mass transport in the system. Simulation results clearly illustrate the observed phenomenon and agree well with experimental data. Employing this simulation for different geometries and operating conditions could assist designing heat transfer devices for various applications such as microreactors and cooling devices for electronics. 


\section{Acknowledgements}

This work was performed in part at the Queensland Node of the Australian National Fabrication Facility, a company established under the National Collaborative Research Infrastructure Strategy to provide nano- and microfabrication facilities for Australia's researchers. The authors acknowledge funding support from Australian Research Council through the ARC linkage grant (LP150100153).

\section{References}

[1] C.T. Nguyen, F. Desgranges, G. Roy, N. Galanis, T. Maré, S. Boucher, H. Angue Mintsa, Temperature and particle-size dependent viscosity data for water-based nanofluids - Hysteresis phenomenon, International Journal of Heat and Fluid Flow, 28 (2007) 1492-1506.

[2] M. Kole, T.K. Dey, Viscosity of alumina nanoparticles dispersed in car engine coolant, Experimental Thermal and Fluid Science, 34 (2010) 677-683.

[3] Y. He, Y. Jin, H. Chen, Y. Ding, D. Cang, H. Lu, Heat transfer and flow behaviour of aqueous suspensions of $\mathrm{TiO} 2$ nanoparticles (nanofluids) flowing upward through a vertical pipe, International Journal of Heat and Mass Transfer, 50 (2007) 2272-2281.

[4] L.S. Sundar, M.K. Singh, A.C.M. Sousa, Thermal conductivity of ethylene glycol and water mixture based Fe3O4 nanofluid, International Communications in Heat and Mass Transfer, 49 (2013) $17-24$

[5] M.T. Naik, G. Ranga Janardhana, K. Vijaya Kumar Reddy, B. Subba Reddy, Experimental investigation into rheological property of copper oxide nanoparticles suspended in propylene glycolwater based fluids, Journal of Engineering and Applied Sciences, 5 (2010) 29-34.

[6] G.P. Peterson, C.H. Li, Heat and mass transfer in fluids with nanoparticle suspensions, Advances in Heat Transfer, 2006, pp. 257-376.

[7] M. Lomascolo, G. Colangelo, M. Milanese, A. De Risi, Review of heat transfer in nanofluids: Conductive, convective and radiative experimental results, Renewable and Sustainable Energy Reviews, 43 (2015) 1182-1198.

[8] A. Huminic, G. Huminic, C. Fleaca, F. Dumitrache, I. Morjan, Thermal conductivity, viscosity and surface tension of nanofluids based on FeC nanoparticles, Powder Technology, 284 (2015) 78-84. [9] G. Huminic, A. Huminic, Heat transfer characteristics of a two-phase closed thermosyphons using nanofluids, Experimental Thermal and Fluid Science, 35 (2011) 550-557.

[10] S. Hassani, R. Saidur, S. Mekhilef, A. Hepbasli, A new correlation for predicting the thermal conductivity of nanofluids; using dimensional analysis, International Journal of Heat and Mass Transfer, 90 (2015) 121-130. 
[11] J. Sarkar, P. Ghosh, A. Adil, A review on hybrid nanofluids: Recent research, development and applications, Renewable and Sustainable Energy Reviews, 43 (2015) 164-177.

[12] W.H. Azmi, K.V. Sharma, P.K. Sarma, R. Mamat, S. Anuar, V. Dharma Rao, Experimental determination of turbulent forced convection heat transfer and friction factor with $\mathrm{SiO} 2$ nanofluid, Experimental Thermal and Fluid Science, 51 (2013) 103-111.

[13] M. Hejazian, M.K. Moraveji, A comparative analysis of single and two-phase models of turbulent convective heat transfer in a tube for TiO2 nanofluid with CFD, Numerical Heat Transfer; Part A: Applications, 63 (2013) 795-806.

[14] M. Hejazian, M.K. Moraveji, A. Beheshti, Comparative study of Euler and mixture models for turbulent flow of Al2O3 nanofluid inside a horizontal tube, International Communications in Heat and Mass Transfer, 52 (2014) 152-158.

[15] M.K. Moraveji, M. Hejazian, CFD examination of convective heat transfer and pressure drop in a horizontal helically coiled tube with CuO/Oil base nanofluid, Numerical Heat Transfer; Part A: Applications, 66 (2014) 315-329.

[16] M. Keshavarz Moraveji, M. Hejazian, Modeling of turbulent forced convective heat transfer and friction factor in a tube for Fe 304 magnetic nanofluid with computational fluid dynamics, International Communications in Heat and Mass Transfer, 39 (2012) 1293-1296.

[17] M. Keshavarz Moraveji, M. Hejazian, Natural convection in a rectangular enclosure containing an oval-shaped heat source and filled with Fe3O4/water nanofluid, International Communications in Heat and Mass Transfer, 44 (2013) 135-146.

[18] M. Hejazian, M.K. Moraveji, A. Beheshti, Comparative numerical investigation on TiO2/water nanofluid turbulent flow by implementation of single phase and two phase approaches, Numerical Heat Transfer; Part A: Applications, 66 (2014) 330-348.

[19] A.N. Afifah, S. Syahrullail, N.A. Che Sidik, Natural convection of alumina-distilled water nanofluid in cylindrical enclosure: an experimental study, J. Adv. Res. Fluid Mech. Therm. Sci., 12 (2015) 1-10.

[20] A. Aghaei, H. Khorasanizadeh, G. Sheikhzadeh, M. Abbaszadeh, Numerical study of magnetic field on mixed convection and entropy generation of nanofluid in a trapezoidal enclosure, Journal of Magnetism and Magnetic Materials, 403 (2016) 133-145.

[21] A. Akbarinia, A. Behzadmehr, Numerical study of laminar mixed convection of a nanofluid in horizontal curved tubes, Applied Thermal Engineering, 27 (2007) 1327-1337.

[22] T. Basak, S. Roy, A.R. Balakrishnan, Effects of thermal boundary conditions on natural convection flows within a square cavity, International Journal of Heat and Mass Transfer, 49 (2006) 4525-4535.

[23] A.K. Abdul Hakeem, N. Vishnu Ganesh, B. Ganga, Magnetic field effect on second order slip flow of nanofluid over a stretching/shrinking sheet with thermal radiation effect, Journal of Magnetism and Magnetic Materials, 381 (2015) 243-257. 
[24] H.R. Ashorynejad, M. Sheikholeslami, I. Pop, D.D. Ganji, Nanofluid flow and heat transfer due to a stretching cylinder in the presence of magnetic field, Heat and Mass Transfer/Waerme- und Stoffuebertragung, 49 (2013) 427-436.

[25] T. Hayat, M. Qasim, Influence of thermal radiation and Joule heating on MHD flow of a Maxwell fluid in the presence of thermophoresis, International Journal of Heat and Mass Transfer, 53 (2010) 4780-4788.

[26] N. Rudraiah, R.M. Barron, M. Venkatachalappa, C.K. Subbaraya, Effect of a magnetic field on free convection in a rectangular enclosure, International Journal of Engineering Science, 33 (1995) 1075-1084.

[27] M. Sheikholeslami, R. Ellahi, Three dimensional mesoscopic simulation of magnetic field effect on natural convection of nanofluid, International Journal of Heat and Mass Transfer, 89 (2015) 799808.

[28] M. Goharkhah, M. Ashjaee, M. Shahabadi, Experimental investigation on convective heat transfer and hydrodynamic characteristics of magnetite nanofluid under the influence of an alternating magnetic field, International Journal of Thermal Sciences 99 (2016) 113-124.

[29] M. Goharkhah, A. Salariana, M. Ashjaee, M. Shahabadi, Convective heat transfer characteristics of magnetite nanofluid under the influence of constant and alternating magnetic field, Powder Technology 274 (2015) 258-267.

[30] Y. Xuan, Q. Li, M. Ye, Investigations of convective heat transfer in ferrofluid microflows using lattice-Boltzmann approach, International Journal of Thermal Sciences 46 (2007) 105-111.

[31] R. Azizian, E. Doroodchi, T. McKrell, J. Buongiorno, L.W. Hu, B. Moghtaderi, Effect of magnetic field on laminar convective heat transfer of magnetite nanofluids, International Journal of Heat and Mass Transfer, 68 (2014) 94-109.

[32] M. Goharkhah, M. Ashjaee, Effect of an alternating non uniform magnetic field on ferrofluid flow and heat transfer in a channel, Journal of Magnetism and Magnetic Materials, 362 (2014) 80-89. [33] M. Yarahmadi, H. Moazami Goudarzi, M.B. Shafii, Experimental investigation into laminar forced convective heat transfer of ferrofluids under constant and oscillating magnetic field with different magnetic field arrangements and oscillation modes, Experimental Thermal and Fluid Science, 68 (2015) 601-611.

[34] M. Lajvardi, J. Moghimi-Rad, I. Hadi, A. Gavili, T. Dallali Isfahani, F. Zabihi, J. Sabbaghzadeh, Experimental investigation for enhanced ferrofluid heat transfer under magnetic field effect, Journal of Magnetism and Magnetic Materials 322 (2010) 3508-3513.

[35] A. Ghofrani, M.H. Dibaei, A. Hakim Sima, M.B. Shafii, Experimental investigation on laminar forced convection heat transfer of ferrofluids under an alternating magnetic field, Experimental Thermal and Fluid Science 49 (2013) 193-200. 
[36] M. Asfer, B. Mehta, A. Kumar, S. Khandekar, P.K. Panigrahi, Effect of magnetic field on laminar convective heat transfer characteristics of ferrofluid flowing through a circular stainless steel tube, International Journal of Heat and Fluid Flow, 59 (2016) 74-86.

[37] M. Hejazian, N.-T. Nguyen, Negative magnetophoresis in diluted ferrofluid flow, Lab on a Chip, 15 (2015) 2998-3005.

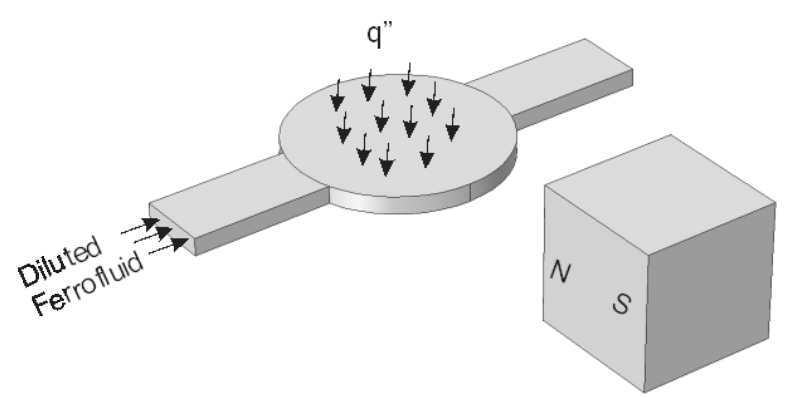

Fig. 1. The system under investigation. 
(a)

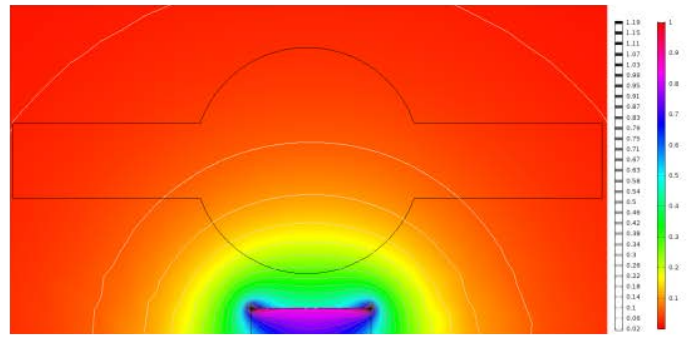

(b)

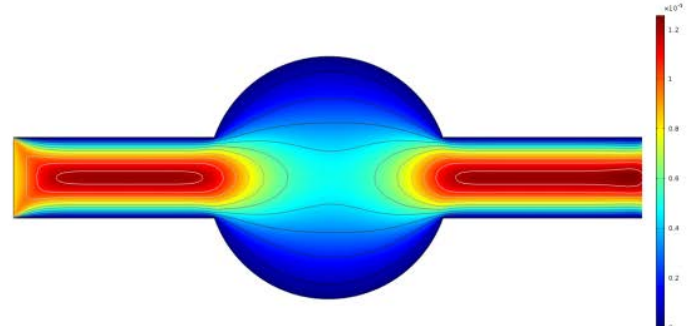

도

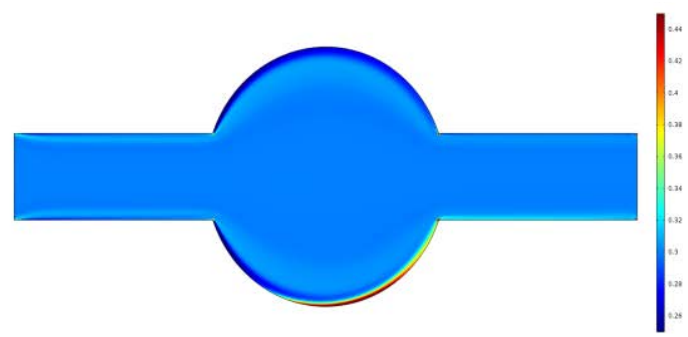

(d)

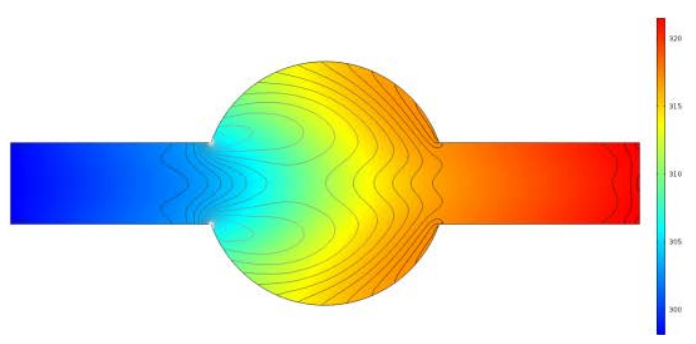

Fig. 2. Simulation results for flow rate of $50 \mu L / \mathrm{min}$, and under magnetic field: (a) Magnetic field distribution; (b) Velocity field; (c) Concentration distribution; (d) Temperature profile. 

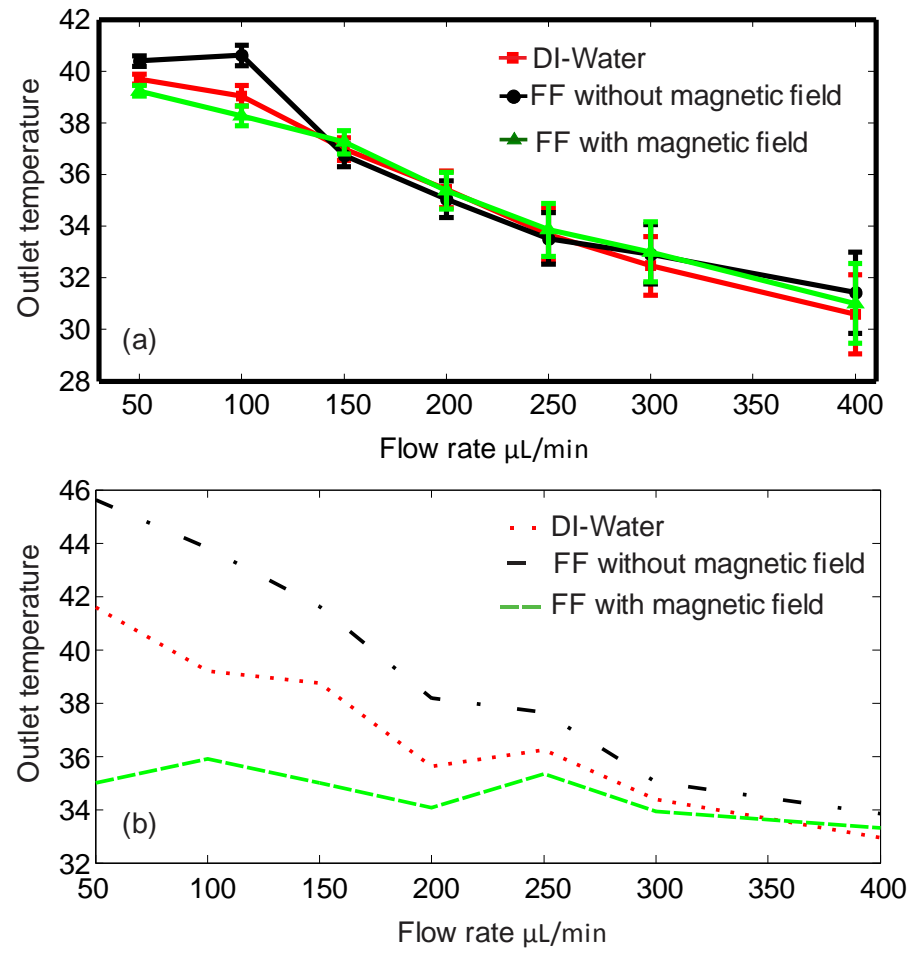

Fig. 3. Outlet temperature: (a) Experimental data; (b) Simulation results. 

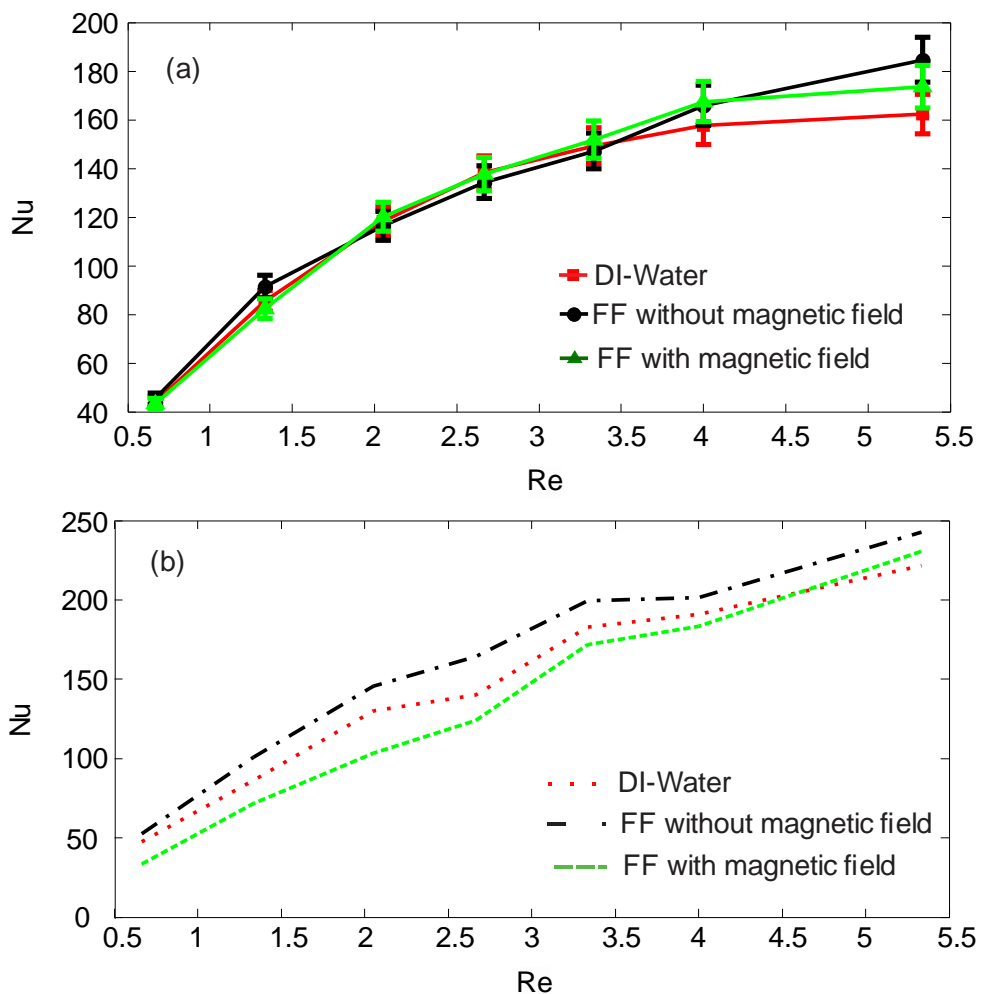

Fig. 4. Nusselt number versus Reynolds number: (a) Experimental data; (b) Simulation results. 